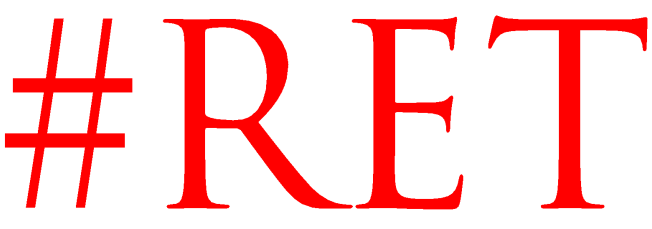

Revista Española de la Transparencia

Núm. 7. Segundo Semestre 2018

ISSN 2444-2607. Págs. 71-97

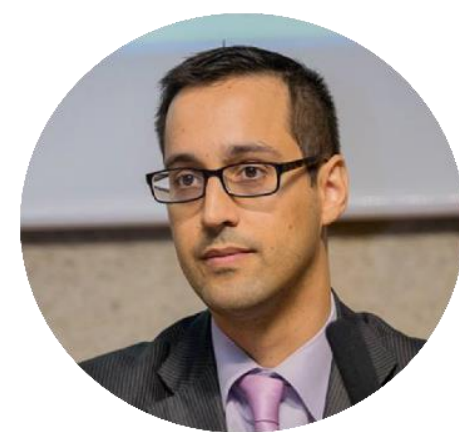

Javier Sierra Rodriguez Universidad de Murcia ${ }^{1}$

\title{
Mediciones y premios de transparencia
}

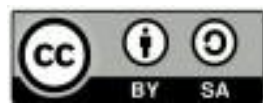

RECIBIDO: 27 de noviembre de 2018 ACEPTADO: 31 de diciembre de 2018

RESUMEN: Los sistemas de medición del grado de transparencia de las entidades públicas cuentan con metodologías y características dispares. En su inicio fueron una palanca esencial para el impulso a la transparencia, pero hoy en día se acusa su falta de adaptación a los avances que se están produciendo, su debilidad metodológica y los problemas que origina la coexistencia de sistemas diferentes.

Este artículo evidencia los problemas actuales de las mediciones de la transparencia y propone que el Consejo de la Transparencia y Buen Gobierno (CTBG) tome una mayor iniciativa para impulsar y ordenar las mediciones en cooperación con el resto de Consejos y Comisionados de la Transparencia y con las organizaciones de la sociedad civil.

PALABRAS CLAVE: transparencia, indicadores, evaluación, mediciones.

CONTENIDOS: 1. Introducción. - 2. Las mediciones de transparencia en España. 2.1. Caracterización de las mediciones. 2.2. Principales mediciones de transparencia. 2.3. La metodología MESTA. 2.4. El Índice de Transparencia de Canarias. 2.5. El Informe anual del Sindic de Greuges de Cataluña. - 2.6. La aportación al avance de la transparencia. - 3. Problemas y debilidades de las mediciones. 3.1. Sobre las entidades que las desarrollan. 3.2. Debilidades metodológicas. 3.3. Consecuencias derivadas. - 4. Reflexión final. - Bibliografía.

\footnotetext{
${ }^{1}$ Doctor en Derecho, Licenciado en Ciencias Políticas y de la Administración, Diplomado en Gestión y Administración Pública por la Universidad de Murcia. Experto en Formador de Formadores en Transparencia por la UNED. Acreditado como PCD. Es profesor asociado en la Universidad de Murcia, Secretario Académico de la Revista Española de la Transparencia y miembro del Comité Organizador del Congreso Internacional de Transparencia desde su primera edición.
} 


\section{Javier Sierra Rodríguez}

\section{Measurements and transparency awards}

ABSTRACT: The measure systems of the transparency grade of the Public Administrations count with different methodologies and characteristics. Initially they were an essential lever to the impulse towards the transparency, but nowadays its lack of adaptation is attributed to the progresses that are taken place, its methodologic weakness and the problems originated by the coexistence of different systems.

This paper highlights the current problems of the transparency measurements and it proposes that the Transparency Council and Good Government takes a major initiative to impulse and order the measurements cooperating with the rest of Councils and Commissioners of the Transparency and with the organizations of the civil society.

KEYWORDS: transparency, indicators, evaluation, measures. 


\section{Mediciones y premios de transparencia}

\section{INTRODUCCIÓN}

¿Qué es una entidad transparente? ¿Qué nivel de transparencia tiene mi organización? Son preguntas cuya respuesta se busca por muchas organizaciones. Frecuentemente para validar el esfuerzo realizado en esta materia, o la por inquietud del responsable político que desea rentabilizar el estereotipo positivo que conlleva este tipo de reconocimientos.

En el presente artículo se pretende realizar un repaso a la situación y a las principales ventajas e inconvenientes de las mediciones de transparencia en España $^{2}$, tanto del desarrollo de metodologías, como de las que se han materializado a través de informes o rankings, ampliando así un primer trabajo realizado años atrás sobre esta misma cuestión (Delgado, López y Sierra, 2015). Debido a la disparidad existente entre las diversas experiencias que se llevan a cabo, se hará referencia indistintamente como mediciones para hacer alusión a todo ejercicio que permita una valoración del nivel de transparencia de las organizaciones.

El objetivo que se pretende cubrir con esta contribución es dar una visión del estado actual de las mediciones de la transparencia, resaltar sus aspectos positivos y negativos, así como ofrecer una serie de planteamientos encaminados a su mejora. Para ello se han examinado las metodologías existentes y se ha analizado su trayectoria en los últimos años, realizando a su vez una amplia revisión de la literatura existente. Cabe mencionar que algunas observaciones que se hacen constar a lo largo del artículo, derivan de un contraste continuo de opiniones de expertos, técnicos, activistas y profesionales que se han recogido en los últimos años sobre las evaluaciones y mediciones de la transparencia.

\section{LAS MEDICIONES DE TRANSPARENCIA EN ESPAÑA}

Antes de la oleada de preocupación por la transparencia, que se intensificó a partir de la publicación de la Ley 19/2013, de 9 de diciembre, de transparencia, acceso a la información pública y buen gobierno (LTBG), ya existían mecanismos para su medición, aunque fue especialmente a partir de su aprobación cuando eclosionaron los informes y análisis comparativos de todo tipo. A continuación se ofrece una visión general sobre ellos, a través de la descripción de los principales instrumentos o, al menos, de los más conocidos que existen en la actualidad.

2 Para conocer experiencias de otros paises, se puede consultar el documento elaborado por Scrollini (2012) para el Consejo de la Transparencia de Chile. Aparte, la Red de Transparencia y Acceso a la Información (RTA) está desarrollando un proyecto para la medición y cuantificación de los avances en la materia de diversos paises que forman parte de esta red (De la Fuente, 2015). 


\section{Javier Sierra Rodríguez}

\subsection{Caracterización de las mediciones.}

Las mediciones más populares suelen desarrollar rankings que tienen un amplio eco en los medios de comunicación social y que citan especificamente la posición y puntuación de cada una de las entidades que son objeto de medición. En un segundo plano -en lo que respecta a repercusión social-, se encuentran los informes que buscan diagnosticar de manera más general los niveles y/o evolución de la transparencia, presentando datos agregados sin indicar especificamente los resultados por entidad.

Las mediciones de la transparencia se han planteado y desarrollado por entidades de diversa naturaleza, tanto públicas como privadas, destacando las organizaciones no gubernamentales, los grupos de investigación de las universidades y los organismos públicos que ejercen algún tipo de actividad en relación a la transparencia.

Las organizaciones objeto de análisis no tienen por qué limitarse a las Administraciones Públicas, sino que también pueden abarcar instituciones y otro tipo de entidades del sector público, partidos políticos, fundaciones y otras tipologías, siempre que sobre ellas se proyecte algún tipo de interés en relación a la transparencia.

Algunas iniciativas han supuesto un mero ejercicio de desarrollo intelectual con el diseño de metodologías que no se han aplicado, mientras que otras se han desarrollado y materializado en informes periódicos y con cierta continuidad en el tiempo.

A su vez, las mediciones pueden variar en la definición de su objeto de estudio, según se busque medir la transparencia de un tipo concreto de organizaciones (Comunidades Autónomas, Universidades, Diputaciones, etc.), o bien, establecer criterios de selección adicional (grandes ayuntamientos, etc.). En otros casos se combinan estos criterios con la condición de que las organizaciones estén ubicadas en un ámbito territorial, siendo frecuentes los estudios que se desarrollan en torno a las entidades de una Comunidad Autónoma.

Las metodologías también son dispares, aunque prácticamente todas siguen un mecanismo similar que chequea el cumplimiento de indicadores prediseñados, cuyo número varía sustancialmente entre ellas. Algunas dan a conocer los indicadores a medir de manera anticipada, permitiendo que las organizaciones que van a ser medidas sepan qué deben cumplir y se preparen para ello; mientras que otras, realizan las mediciones y publican los resultados directamente y sin previo aviso. 


\section{Mediciones y premios de transparencia}

Dichas mediciones se pueden realizar por la propia entidad evaluadora a través de la información disponible, generalmente en las páginas web, o bien tener algún tipo de interactuación con las organizaciones cuya transparencia se quiere medir. Así, por ejemplo, es habitual partir de un cuestionario autocumplimentado cuyos contenidos son verificados posteriormente.

De ahí, que algunas limitan la medición a las entidades que responden a dichos cuestionarios o que hayan satisfecho una solicitud de información previa, publicando a su vez la relación de organizaciones no colaboradoras como fórmula de evidenciar la falta de implicación en los estudios. En otras ocasiones, la limitación del número de entidades a medir deriva de su alto número -lo que haría inviable abarcarlas a todas-, como por ejemplo, ocurre con los Ayuntamientos españoles. En estos casos, algunas permiten que las entidades interesadas soliciten su inclusión. De este modo, el rango de posibilidades es muy amplio según el ranking o informe concreto del que se trate.

\subsection{Principales mediciones de transparencia.}

El repaso a las principales mediciones de transparencia lleva a los informes e indices desarrollados por organizaciones no gubernamentales y grupos de investigación de Universidades, así como a las metodologías y análisis desarrollados por instituciones públicas. Por razones de espacio no se incluyen las referencias a los numerosos estudios y análisis de carácter puntual y vocación científica que se han realizado por académicos, bien a través de mediciones propias, o precisamente basados en los indicadores o/y resultados de mediciones preexistentes, tratando de establecer conclusiones generalizables ${ }^{3}$.

\section{a. Experiencias previas a la LTBG.}

Transparencia Internacional fue una de las pioneras en 2008 con la medición de entidades públicas a través del Índice de Transparencia de los Ayuntamientos (Ros, 2018: 5), al igual que otras organizaciones como la Fundación Compromiso y Transparencia, que se centró inicialmente en las organizaciones no

\footnotetext{
${ }^{3}$ A modo de ejemplo se pueden citar algunos que proyectan sus análisis sobre la transparencia fiscal (Caamaño, Lago, Reyes y Santiago, 2011; Esteller y Polo, 2012), la explicación de los niveles de transparencia en contextos de crisis (Tejedo y Ferraz, 2015), el cumplimiento de las obligaciones de transparencia y reutilización en la contratación pública de la AGE (Beltrán y Martínez, 2016), las webs de las Comunidades Autónomas (García y Curto, 2018), la transparencia en los municipios españoles (Villoria e Iglesias, 2017; Delgado, Navarro y Mora, 2017; Mora, Delgado y Rivero, 2018), de los ayuntamientos de Asturias (Alonso y García, 2014; García, Alonso y Alonso, 2017), de Castilla La Mancha (Manfredi et al. 2016) y de Cataluña (Molina, Rodríguez, Corcoy y Vadillo, 2017), de los partidos políticos (Martínez Isidoro, 2017; Pintado, 2018; Díez, 2017), o de cuestiones mucho más concretas como los operadores de gestión/explotación de redes de transporte público (Barqueros, 2018), entre muchos otros.
} 


\section{Javier Sierra Rodríguez}

gubernamentales publicando en 2009 el primer informe de transparencia y buen gobierno de las fundaciones españolas ${ }^{4}$.

A partir de esa fecha, ambas organizaciones fueron ampliando el elenco de mediciones. Transparencia Internacional España ha publicado periódicamente índices centrados en organizaciones, tanto públicas como privadas, abarcando los Ayuntamientos (ITA), las Comunidades Autónomas (INCAU), las Diputaciones provinciales (INDIP), las organizaciones de gestión de recursos hídricos en el que incluyen Confederaciones Hidrográficas y entidades de gestión del agua (INTRAG), los Parlamentos (IPAR), los Clubes de Fútbol (INFUT), las Federaciones Deportivas (INFED), las Empresas Públicas (INDEP), así como los partidos políticos. Sin embargo, a fecha de cierre de este artículo parece existir un parón en la actividad de medición porque algunos de los índices no han repetido su edición en 2018.

Por su parte, la Fundación Compromiso y Transparencia (antes denominada Compromiso Empresarial) fue elaborando informes sobre la transparencia en medios de comunicación y en museos (2010), partidos políticos y universidades (2012), Consejos de Administración de las empresas del IBEX35 (2013), empresas farmacéuticas (2017), además de otros informes relacionados con la independencia de las organizaciones y su grado de credibilidad.

En paralelo a estas etapas iniciales, se fue gestando el Mapa Infoparticipa por iniciativa de investigadores de la Universidad Autónoma de Barcelona. A partir de un proyecto de investigación relacionado con la comunicación en los entornos locales (Moreno Sardà et al., 2013). En 2012 dio un giro hacia la detección de buenas prácticas de comunicación pública local, evolucionando a lo que hoy conocemos por su vertiente de medición de la transparencia, junto con el sello Infoparticipa que otorga a las mejores web municipales que cumplen los requisitos establecidos por su sistema.

\section{b. Experiencias posteriores a la LTBG.}

Tras la publicación de la LTBG surgieron nuevas iniciativas de medición y evaluación de la transparencia. Así, entre las primeras de este nuevo periodo se diseñó el Sistema Español de Acreditación de la Transparencia, de la actual Asociación de Profesionales e Investigadores de la Transparencia (ACREDITRA). El sistema, que se concebía como un ensamblaje consultores, auditores y comisión

\footnotetext{
4 En el ámbito de las ONGs existen mecanismos que tienen un mayor recorrido y que están orientados para dotar de credibilidad a su actuación y de una mayor confianza a sus financiadores (Gálvez, Caba y López, 2009), entre ellos los sistemas de la Fundación Lealtad o de la Coordinadora de ONGs para el Desarrollo, los cuáles no se tratan en este trabajo al focalizar su atención en los sistemas orientados a las organizaciones de carácter público y bajo el marco de la LTBG.
} 


\section{Mediciones y premios de transparencia}

externa de control (Sierra, 2014), nunca se llegó a poner en marcha más allá de su experiencia piloto ${ }^{5}$.

Al igual que la iniciativa descrita, surgieron otras, como el informe "Test de aplicación de la Ley de Transparencia", desarrollado por el Departamento de Economía de la Empresa de la Universidad Rey Juan Carlos y que publica sus informes en el portal SIELOCAL, abarcando los Ayuntamientos (2014 y 2017) y las Diputaciones españolas en 2015, entre otras iniciativas de medición que superan el ámbito de la transparencia.

También existe el índice Dyntra, que realiza mediciones sobre un amplio elenco de entidades (universidades, diputaciones y ayuntamientos, entre otras) y que tiene mucha difusión e impacto en medios de comunicación, estando muy enfocado al premio en términos de reputación.

Aparte hay muchas otras experiencias de alcance más limitado, por suponer metodologías que apenas se han aplicado o carecen de una frecuencia determinada ${ }^{6}$, abarcar solo a las entidades de un territorio concreto -generalmente respecto a una Comunidad Autónoma ${ }^{-7}$, o por ceñirse a tipologias especificas de organizaciones públicas ${ }^{8}$. De ellas, se analizarán a continuación el sistema MESTA del CTBG y las iniciativas del Sindic de Greuges de Cataluña y del Comisionado de la Transparencia de Canarias.

\subsection{La metodología MESTA.}

El Consejo de la Transparencia y Buen Gobierno (CTBG) tiene entre sus funciones evaluar el grado de aplicación de la LTBG (art. 38.1.d LTBG), por lo que ha elaborado diversos informes de evaluación sobre el cumplimiento de las obligaciones de la Ley del portal de transparencia de la AGE, de los órganos constitucionales y reguladores, o sobre las entidades que configuran el Sector Público de la Administración General del Estado?

\footnotetext{
5 Acreditra es la organización que actualmente promueve la Revista Española de Transparencia y el Congreso Internacional de Transparencia, que ya va por su tercera edición.

6 Entre ellas, el Grupo de Investigación PsiCom de la Universidad de Santiago de Compostela desarrolló la Metodología de Gestión de la Transparencia Pública (MGT-GAL) que contemplaba un sistema de acreditación y un sello de transparencia.

7 La Cámara de Cuentas de Andalucía en colaboración con la Universidad de Granada y la Fundación Centro de Estudios Andaluces ha desarrollado una metodología para medir la transparencia en el ámbito municipal (Zafra y Garrido, 2017).

8 Analítica Pública destaca por haber realizado mediciones sobre la transparencia en las universidades, así como análisis innovadores sobre cuestiones de diseño y navegación en los portales de transparencia.

9 Véanse las secciones correspondientes a los informes y documentación de la web del Consejo de Transparencia: www.consejodetransparencia.es
} 


\section{Javier Sierra Rodríguez}

A partir de un convenio entre el CTBG y la anterior Agencia Estatal de Evaluación de las Políticas Públicas y la Calidad de los Servicios (AEVAL) ${ }^{10}$ se puso en marcha el diseño de una metodología de evaluación y seguimiento de la transparencia de la actividad pública denominada MESTA (Arizmendi, 2017) ${ }^{11}$.

El sistema abarca los dos pilares básicos de la transparencia: la publicidad activa y el derecho de acceso. Respecto a la publicidad activa, su enfoque contempla la medición de la información publicada (contenidos, forma y actualización), de los atributos de dicha información (accesibilidad, claridad, estructuración y reutilización) y del soporte web que la alberga (accesibilidad, estructuración, lugar de publicación y disponibilidad de banner). Tiene en cuenta lo que denomina el mapa de publicidad activa de cada organismo, que diferencia entre la información obligatoria según la LTBG y el resto de información según sea aplicable a la entidad concreta sobre la que se va a medir la transparencia.

\section{INDICADORES DE PUBLICIDAD ACTIVA DE MESTA}

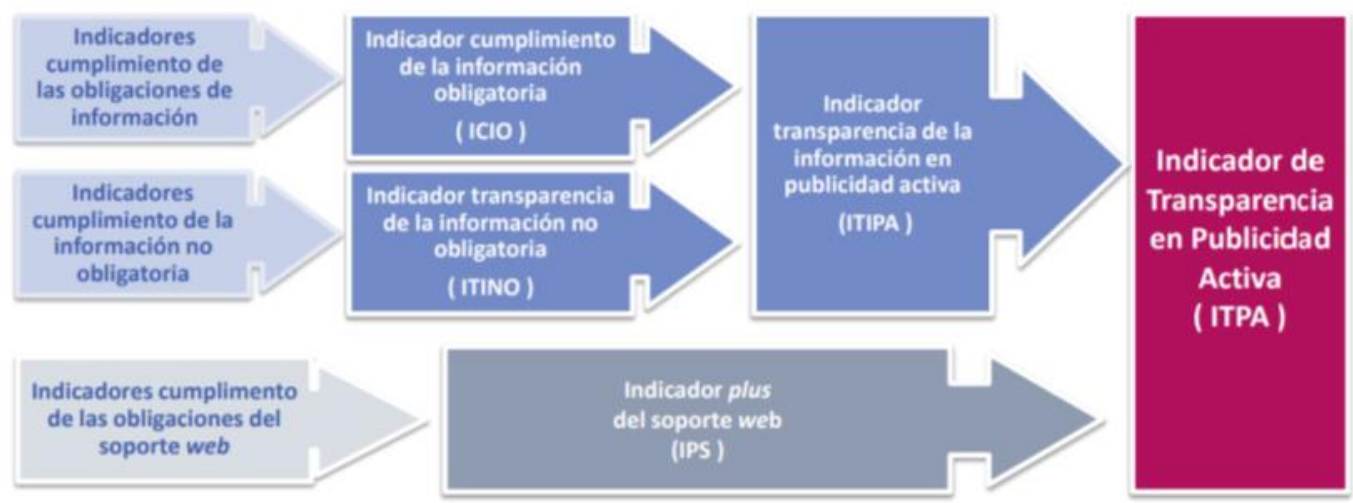

Fuente: Informe final MESTA. AEVAL 2016.

Para su desarrollo prevé un cuestionario autocumplimentado de actividades, a partir del cual se caracteriza a la organización para definir el mapa de publicidad activa al que está sometido. Un segundo cuestionario está destinado a obtener la información que será objeto de valoración.

Para ello, utiliza el Indicador de Cumplimiento en Publicidad Activa (ICPA) que se basa, tanto en la información publicada, como en sus atributos y en las condiciones técnicas del soporte web. El sistema tiene un segundo Indicador de Transparencia en Publicidad Activa (ITPA) que básicamente incluye los anteriores y, además, considera la información no obligatoria y las funcionalidades del soporte web que

10 Actualmente disuelta por el Real Decreto 769/2017, de 28 de julio, y cuyas funciones se han asumido por la Secretaría de Estado de Función Pública.

11 Véase el informe MESTA. Metodología de Evaluación y Seguimiento de la Transparencia de la Agencia Estatal de Evaluación de las Políticas Públicas y la Calidad de los Servicios fechado el 30 de junio de 2016. 


\section{Mediciones y premios de transparencia}

superan los mínimos de la LTBG. La metodología recoge recomendaciones adicionales como la realización de reconocimientos de buenas prácticas, la certificación de la transparencia, entre otras.

Por otra parte, MESTA incluye la medición de aspectos relativos al derecho de acceso. Para ello estructura las mediciones distinguiendo entre tres fases: ejercicio del derecho, tramitación y finalización. Prevé la utilización de tres cuestionarios que incluyen preguntas o contenidos para cada una de las fases: un cuestionario de autoevaluación para la valoración de caracteristicas, una ficha resumen y una ficha de evidencias que sustentan las valoraciones. La metodología establece una serie de recomendaciones para complementar el sistema y validar la información, entre las que se encuentra la realización de valoraciones externas, de encuestas a los usuarios o la aplicación de la técnica del cliente misterioso.

Pese a este diseño tan completo, el sistema MESTA no ha sido puesto en práctica de manera generalizada y se ha aplicado fragmentariamente en los informes antes mencionados, además de ser la referencia utilizada para la experiencia piloto desarrollada por el Consejo de la Transparencia de la Región de Murcia y de las mediciones periódicas que lleva a cabo el Comisionado de la Transparencia de Canarias.

De este modo, la función de evaluación se ha visto relajada y la proactividad y liderazgo que seria esperable en esta materia por el CTBG no se está produciendo, motivo por el que otras organizaciones han ocupado parte del espacio público que correspondería por naturaleza al CTBG. A ello contribuye la debilidad actual del CTBG, porque no se ha nombrado un nuevo titular del órgano -cuya presidencia se ejerce en funciones actualmente-, y las restricciones presupuestarias que ha sufrido (Sánchez de Diego, 2018:11) $)^{12}$.

\subsection{El Índice de Transparencia de Canarias.}

Por su parte, el Comisionado de la Transparencia de Canarias puso en marcha en 2016 la plataforma "T-Canarias"13, que hace posible el índice de Transparencia de Canarias (ITCanarias) para la medición de la transparencia de ayuntamientos, cabildos, Gobierno de Canarias y universidades. Dicha plataforma permite a las entidades objeto de medición incluir la información relativa a los dos índices que lo componen: el indicador de cumplimiento de la publicidad activa (ICPA), que mide principalmente el cumplimiento de las obligaciones legales en la materia; y el indicador de transparencia voluntaria (ITV), que comprueba aquellas prácticas que

12 La falta de recursos del CTBG y de los organismos de control de la transparencia de las Comunidades Autónomas ha sido objeto de una de las reclamaciones incluidas en la Declaración de Cádiz de la Red de Consejos y Comisionados de la Transparencia, suscrita el pasado septiembre de 2018, y apoyada por más de una treintena de organizaciones de la sociedad civil.

13 El acceso a la aplicación y a sus manuales de utilización están accesibles en la dirección: https://sede.transparenciacanarias.org/publico/evaluacion 


\section{Javier Sierra Rodríguez}

superan las obligaciones legales. El ICPA a su vez contempla dos vertientes, por un lado las características de la información y el modo de su publicación, y por otro, las caracteristicas del soporte web. Para todo el proceso utiliza tres cuestionarios, uno de carácter general, un cuestionario de estadísticas sobre el derecho de acceso y un cuestionario de publicidad activa.

Al final es un sistema que no se limita solo a medir la existencia de información, sino también las características del modo en que están publicadas y las funcionalidades del sitio web, contemplando aspectos como la actualización de contenidos, la accesibilidad, la reutilización, la estructura de contenidos o la disposición de un buscador. Entre las ventajas que muestra este sistema, está su estandarización e implementación a través de una plataforma tecnológica que permite el cálculo automatizado de los resultados gracias a un algoritmo, además de la sistematización y especificación en detalle que se hace de los criterios de puntuación y valoración. Por el contrario, la necesidad de automatización y estandarización puede suponer una limitación a la hora de captar fielmente el grado de calidad de la información y de los sitios web que solo se podrian determinar mediante la intervención humana.

\subsection{El informe anual del Sindic de Greuges de Cataluña.}

El Sindic de Greuges, confecciona anualmente un informe sobre la transparencia centrado en las entidades públicas de Cataluña. En sus informes no se presentan los resultados desagregados por entidad, pero se incluyen públicamente las bases de datos con los registros individuales que permiten su explotación por cualquier interesado.

Entre las fortalezas e innovaciones que aporta este informe en su edición de 2018, se incluía una valoración cualitativa de la información publicada de una selección de entidades y la medición de la percepción de setenta ciudadanos tras la experiencia de búsqueda de información. En ambos casos, el número de entidades sobre los que se proyectaban dichas mediciones era mínimo (12 y 16 entidades respectivamente), pero se considera una buena práctica porque puede ser una experiencia piloto generalizable o exportable a otras mediciones.

Además, este informe recogía la medición de la situación respecto al derecho de acceso mediante dos fórmulas. Por un lado, utilizando el test del ciudadano oculto se realizaron 228 solicitudes de acceso a la información pública, con el propósito real de medir la capacidad de respuesta de las entidades. La segunda fórmula consistió en el análisis de cien resoluciones desestimatorias de solicitudes publicadas por la Administración de la Generalitat de Catalunya y del Ayuntamiento de Barcelona. 


\section{Mediciones y premios de transparencia}

Este informe se muestra como una experiencia destacable, que rebasa el concepto de medición y que podría insertarse en un concepto más amplio de evaluación de la transparencia. De hecho, el informe anual no se limita a una exposición descriptiva de resultados, sino que aborda adecuadamente unas conclusiones y formula recomendaciones de mejora.

\subsection{La aportación al avance de la transparencia.}

Las evaluaciones de transparencia han ejercido un importante papel para introducir esta materia en las agendas políticas de los Gobiernos, producir competencia entre las entidades públicas por ser más transparentes ${ }^{14}$, y hacer que se produzcan importantes avances en la publicación de información y documentación en línea, ya fuese por convencimiento o por temor a quedar en evidencia por una mala puntuación en las mediciones. Así, Sánchez de Diego (2014: 17) indicaba en relación al ITA, que "lo más sobresaliente de esta acción es la reacción generalizada de los Ayuntamientos que buscan mejorar su posición en el ranking", a lo que contribuye como destacan Alonso y Garcia (2014) el "gran impacto mediático que presiona a los gestores públicos a volcar sus esfuerzos en él".

Evidentemente, no solo los rankings han impulsado esta dinámica sino que, además, ha contribuido una conjunción de factores como las obligaciones de la ley, el papel de los medios de comunicación, la sociedad civil o el propio impulso de las entidades que debian implementarla. No obstante, es cierto que los rankings destinados a medir la transparencia han provocado un mayor escrutinio sobre las organizaciones objeto de medición, y que una mala puntuación ha servido en muchos casos para estimular que se pongan al día. De hecho, quienes han trabajado en contacto con las áreas de transparencia de los principales Ayuntamientos, de las Diputaciones, los Parlamentos o las Comunidades Autónomas, habrán podido comprobar la expectativa y preocupación de los técnicos y de los responsables políticos ante la inminente publicación de los rankings de referencia.

De ahí que al observar evoluciones positivas, pueda otorgarse un mayor mérito a los rankings en ese contexto. Aun cuando se ven afectadas por otros factores, como el contexto normativo, se presume que han actuado como una palanca para activar mejoras en materia de transparencia. Por citar algunos ejemplos, en el caso del INCAU, se pasó de una media de cumplimiento de 71,5 puntos sobre 100 en 2010, a 94 de media en 2016. Algo similar ocurrió con el ITA, que en su edición 2008 arrojaba una media de 52,1 sobre 100, para llegar a 89,7 en 2017. Se observa que

14 Este rasgo ha sido buscado conscientemente por algunos sistemas de evaluación y se indicaba como uno de los objetivos perseguidos por el Mapa Infoparticipa con la visualización automática de los resultados, caracteristica que se complementa con la difusión de los resultados a través de medios de comunicación y con el otorgamiento del Sello Infoparticipa (Moreno, Molina y Simelio, 2017:374). 


\section{Javier Sierra Rodríguez}

suben en puntuación las entidades que son objeto de medición, pero además, algunos estudios empíricos apuntan a que los municipios sobre los que no se mide la transparencia tienen un nivel de transparencia mucho menor (Alonso y Garcia, $2014)^{15}$

\section{PROBLEMAS Y DEBILIDADES DE LAS MEDICIONES}

El problema de partida respecto a las mediciones de transparencia es que no existe un estándar al respecto, de modo que cada entidad aplica los criterios que estima convenientes. Es cierto que hay unos mínimos de contenido que suelen incluirse y que corresponden con la relación de información objeto de publicidad activa que establece la LTBG (arts. 6 a 8), pero no hay una metodología plenamente aceptada y ni siquiera la iniciativa MESTA del CTBG parece haber tenido una fuerte influencia en esta línea.

Tampoco existe una regulación al respecto, salvo las atribuciones que puedan tener y ejercer eventualmente los organismos de control de la transparencia, aunque precisamente la insuficiencia de la LTBG y la lentitud con la que se están abordando cuestiones pendientes como la aprobación de su reglamento, hace deseable que las mediciones de transparencia no sean parte de su objeto, porque se encontrarian ante el riesgo cierto de quedar desfasadas en poco tiempo. No obstante, desde ámbitos relacionados con el control externo, se aboga por establecer obligaciones para la realización de auditorías en función del tipo de entidad pública ${ }^{16}$, así como por regular el papel que deben desempeñar las firmas privadas en este proceso (Viñas, 2018).

Eso no impide que haya sistemas de valoración que se consideren referentes, bien porque tengan éxito respecto a su consideración mediática, o porque son puestos en valor y defendidos como instrumentos válidos principalmente por los "evaluados" que se ven beneficiados por un buen posicionamiento. En cualquier caso, ninguna medición es aceptada y considerada como un instrumento completamente válido. Además, tienen múltiples problemas que impiden el reconocimiento consensuado de todo el ecosistema de actores que trabajan en relación a la transparencia, aunque las voces críticas apenas se escuchen abiertamente en los foros relacionados con la materia.

La lista de problemas de las mediciones es muy amplia y, sin ánimo de ser exhaustivos, a continuación se expondrán algunos de ellos en torno a tres ámbitos:

15 No obstante, esta afirmación debe matizarse porque en el mismo estudio y como observaban sus autores, se detectaba cierta correlación entre el tamaño poblacional del municipio y los avances en materia de transparencia, y son precisamente los municipios pequeños los que no suelen estar incluidos en las mediciones del ITA.

16 Autores como Giménez-Chornet (2017) abogan por la utilización de las auditorias como herramienta que capta con mayor fidelidad la situación respecto a la transparencia en las organizaciones, porque va más allá de la información publicada en internet. 


\section{Mediciones y premios de transparencia}

a) problemas relacionados con las entidades que realizan mediciones, b) problemas de tipo metodológico y c) consecuencias contraproducentes de las mediciones.

\subsection{Sobre las entidades que las desarrollan.}

Hasta el momento se ha dejado entrever que se echa de menos un mayor liderazgo en las evaluaciones por parte de los organismos de control de la transparencia, y específicamente del CTBG, quien por su naturaleza estaría llamado a ejercerlo con unas mayores garantías en lugar de las entidades privadas.

Se ha escrito mucho sobre las carencias en los rasgos de independencia de los organismos de control de la transparencia (Sendín, 2014; Jiménez Asensio, 2017; Martín Delgado, 2018; Sierra, 2018). Sin embargo, no pasa lo mismo respecto a las entidades privadas o de otra naturaleza que realizan los rankings y mediciones que no dejan de ser otra forma de control e incluso de influencia sobre las entidades que son objeto de medición-. Poco o nada se ha dicho sobre esta cuestión que constituye el primer problema que se examina.

En la mayoría de los casos se trata de organizaciones sin fin de lucro o grupos universitarios de investigación, lo que les presupone su independencia. Sin embargo, hay que atender a la finalidad que se busca con la elaboración de las mediciones para determinarlo en mayor medida: si es para cumplir con sus objetivos estatutarios o de producción de conocimiento científico, o si por el contrario, quedan desvirtuadas por la persecución de otras finalidades complementarias. De hecho, hay organizaciones que insisten en la gratuidad de su trabajo en las mediciones como un valor en sí mismo, pero, sin embargo, es evidente que buscan a cambio una recompensa en términos de reputación y aparición en medios de comunicación, cuando no se dedican directamente a la firma de convenios con contenido económico con las mismas entidades públicas sobre las que miden su transparencia, o a prescribir a las empresas con las que tienen relación o son de sus integrantes, por lo que se disipa tal gratuidad, e incluso se detecta cierto conflicto de interés.

En relación a lo anterior, se deduce la necesidad de que sean organizaciones ejemplares e íntegras en su transparencia interna, en su gobernanza y en su manera de actuar -como mínimo tanto como la predican- y cumpliendo los indicadores que ellas mismas exigen para con otros -la transparencia de la transparencia y la integridad de la integridad-. Por el contrario, muchas de las organizaciones que impulsan mediciones no publican unos mínimos respecto a su información corporativa y sus cuentas, así como otra información derivada precisamente de sus tareas de medición, como la explicación en detalle de la metodología aplicada y los recursos destinados para ello, o las posibles relaciones que tienen con las entidades sobre las que miden la transparencia. 
El segundo problema clave que se detecta respecto a las organizaciones que realizan mediciones es la falta de recursos. Es la causa que ha llevado a que las entidades públicas no puedan desarrollar y aplicar los sistemas diseñados en toda su extensión, pero también es el origen de que se produzcan problemas y debilidades manifiestas en las mediciones de las entidades privadas que las llevan a cabo.

Cuando las mediciones dependen de la labor voluntariosa y altruista de los integrantes de organizaciones sin ánimo de lucro, su disposición no siempre es prolongada en el tiempo -abundando la discontinuidad y las mediciones puntuales - y, además, se condiciona el tipo de medición, que tiende a ser sencilla para ahorrar tiempo y esfuerzo. No obstante, esta tendencia también se produce entre las entidades que dedican personal propio o que se nutren del trabajo de becarios.

Los condicionamientos se convierten en un problema cuando se tiende a la excesiva simplicidad, lo que unido a las carencias de recursos, conlleva el riesgo de llevar a cierta falta de rigor o profundidad en las mediciones. Se presume que el trabajo de medición sea honesto y objetivo, pero hay que manifestar que no siempre se alcanzan los estándares necesarios, bien porque la metodología no lo permite, por ejemplo, por la utilización de indicadores que no son unívocos y sobre los que se puedan aplicar criterios dispares; o bien por una aplicación poco profesional, porque no se dispone del personal necesario. El voluntarismo o el hecho de que sean mediciones realizadas por la sociedad civil o que sean gratuitas, no debería atenuar la exigencia de hacer bien las cosas y de salvaguardar la credibilidad en torno a lo que es y lo que significa la transparencia.

\subsection{Debilidades metodológicas.}

Si hablamos de transparencia, lo primero que hay que preguntarse es qué es la transparencia para saber qué ha de medirse. Dicho concepto, sobre el que se manejan múltiples definiciones y sobre el que se puede debatir mucho -cuya discusión no es el objeto de esta contribución-, correspondería a un ideal. Por tanto, el concepto transparencia dependerá de los criterios con los que se evalúa en un contexto y entorno dado, el cual irá evolucionando con el paso del tiempo y exigirá que también se vayan adaptando las mediciones. De hecho, lo que ayer parecia transparente, hoy puede ser insuficiente; mientras que las circunstancias que se consideren transparentes en la actualidad, mañana pueden dejar de serlo.

Aparte, como bien exponen Molina, Simelio y Corcoy (2017: 820 y ss.) las evaluaciones variarán en función del objetivo de evaluación y de la definición de transparencia que se maneje. Así, valorar la transparencia en relación a la corrupción exige incorporar indicadores relativos a la transparencia en materia económica, contratación, etc. Un control desde el punto de vista del cumplimiento de las obligaciones legales llevaría a la medición de aquellas; mientras que un 


\section{Mediciones y premios de transparencia}

enfoque basado en los derechos, debería acudir a indicadores que permitan medir las posibilidades que van más allá del mero cumplimiento legal.

Las mediciones que tienen un mayor impacto no parece que puedan reflejar la transparencia de una organización, entre otras cosas, porque se centran en la información publicada. Podría hablarse de indicadores de publicidad activa transparencia activa- (Rey, 2016), o utilizando un eufemismo, transparencia online, pero sin confundirla con el concepto de transparencia que es más amplio y recoge, como mínimo, el derecho de acceso a la información, que no está presente en la mayoria de los rankings de mayor repercusión mediática ${ }^{17}$.

De ahí que, a la vista de los diferentes indicadores, se observa que no hay una conceptualización alineada adecuadamente con ellos, entre otros motivos, porque los conceptos de los que parten no se definen lo suficiente, o bien, porque se utiliza el concepto de transparencia para mediciones que realmente solo abarcan una parcela de la misma. Esta falta de correspondencia entre los conceptos e indicadores es detectada por Ros (2018: 20), quien subraya que en el caso de Transparencia Internacional sus indicadores no surgen tras un planteamiento metodológico-conceptual; mientras que en relación a la metodología MESTA, apunta a su insuficiente definición teórica, porque se centra en el cumplimiento de las obligaciones de la Ley y no en el nivel de transparencia de las entidades.

En los últimos tiempos, se ha observado ya cierto agotamiento de algunos indicadores, que reflejan esa necesidad de un mayor despliegue en relación a los conceptos de los que parte y que no han evolucionado tan rápido como la realidad. Esta situación ha llevado a que, en algunos casos, las entidades objeto de medición sean todas muy transparentes, cuando es obvio que hay muchos caminos por los que se debe seguir avanzando. El ejemplo más claro es la evolución del INCAU, en cuya última edición de 2016 todas las Comunidades Autónomas tenían puntuaciones superiores a los ochenta puntos sobre los cien que se podian obtener, mientras que catorce de las diecisiete Comunidades Autónomas rebasaban los noventa puntos ${ }^{18}$. El ITA en su edición de 2017 otorgaba la máxima puntuación de cien a veinticinco Ayuntamientos, quedando setenta Ayuntamientos con una puntuación igual o superior a noventa puntos sobre cien.

17 Por ello, se echa de menos la incorporación de indicadores aplicados al derecho de acceso, que recojan experiencias similares a las que se desarrollaban en la iniciativa tuderechoasaber.es de la Fundación Civio y Access Info Europe. Aparte, hay ámbitos relacionados con la transparencia como la reutilización de datos que cada vez es menos justificable que se omitan. En esta línea, Rey (2016) hace referencia a la vertiente de la transparencia colaborativa y expone algunas experiencias internacionales que se centran en ellas como PSI Scoreboard (EPSI platform), Global Open Data Index (OKFN), Open Data City Census (OKFN o el Open Data Barometer (Web Foundation). Véase también la descripción sobre la metodología Meloda que exponen Abellá, Ortiz de Urbina y De Pablos (2014) para evaluar la reutilización de datos abiertos.

18 Es cierto, por otra parte, que Transparencia Internacional ha ido incorporando nuevos indicadores en las últimas mediciones publicadas, pero tal y como se observa, es necesario seguir avanzando en esta linea y posiblemente desechar indicadores que se den por superados. 


\section{Javier Sierra Rodríguez}

ÍNDICE DE TRANSPARENCIA DE LAS COMUNIDADES AUTÓNOMAS

\begin{tabular}{|c|c|c|c|c|}
\hline Año & \multicolumn{2}{|c|}{2010} & \multicolumn{2}{|c|}{2016} \\
\hline $\begin{array}{l}\text { Comunidad } \\
\text { Autónoma }\end{array}$ & Posición & Puntuación & Posición & Puntuación \\
\hline Andalucia & 1 & 87.5 & 7 & 96,3 \\
\hline Aragón & 9 & 70 & 15 & 88,8 \\
\hline Asturias & 1 & 67.5 & 12 & 93,8 \\
\hline Baleares & 15 & 56,3 & 2 & 98,1 \\
\hline Canarias & 12 & 66,3 & 8 & 95 \\
\hline Cantabria & 17 & 53,8 & 8 & 95 \\
\hline Castilla La Mancha & 13 & 62,5 & 14 & 90,6 \\
\hline Castilla y León & 8 & 73,8 & 10 & 94,4 \\
\hline Cataluña & 4 & 82,5 & 2 & 98,1 \\
\hline Extremadura & 5 & 81,3 & 16 & 88,1 \\
\hline Galicia & 1 & 87.5 & 13 & 91,9 \\
\hline La Rioja & 3 & 83,8 & 5 & 97,1 \\
\hline Madrid & 6 & 80 & 2 & 98,1 \\
\hline Murcia & 9 & 70 & 6 & 96.9 \\
\hline Navarra & 6 & 80 & 17 & 81,9 \\
\hline País Vasco & 14 & 57.5 & 1 & 100 \\
\hline Valencia & 15 & 56,3 & 10 & 94,4 \\
\hline
\end{tabular}

Fuente: Transparencia Internacional https://transparencia.org.es

Aun aceptando estas mediciones en su configuración actual, sigue existiendo el problema de que los indicadores se centran primordialmente en la cantidad de información disponible (Ros, 2018: 20) sin abarcar otros posibles criterios. Así, se asimila un nivel de transparencia máxima a ocupar las primeras posiciones de rankings, cuando realmente solo son "una simple comprobación de la publicación de una información en la web" (Delgado, López y Sierra, 2015: 125), que en la mayoría de los casos no tiene en consideración su calidad o el cumplimiento de los principios que establece la LTBG, sin cuya observancia no hay transparencia posible (Garcia Melián, 2017: 331). Por ello conviene recordar el literal del artículo 5.4 LTBG, que realiza un mandato que no se ve reflejado en los indicadores de muchos rankings existentes en España:

«La información sujeta a las obligaciones de transparencia será publicada en las correspondientes sedes electrónicas o páginas web y de una manera clara, estructurada y entendible para los interesados y, preferiblemente, en formatos reutilizables. Se establecerán los mecanismos adecuados para facilitar la accesibilidad, la interoperabilidad, la calidad y la reutilización de la información publicada así como su identificación y localización». 


\section{Mediciones y premios de transparencia}

En definitiva, las mediciones que más se aplican, se centran en qué se publica, pero no en cómo se publica. Se quedan por el camino aspectos de carácter cualitativo que suelen omitirse o cuyo desarrollo es muy tímido, como la facilidad para comprender la información mediante un lenguaje adaptado a los ciudadanos -que no sea excesivamente técnico o jurídico-, la incorporación de elementos visuales que permitan la identificación de los apartados y de la información (iconos, símbolos, negritas, viñetas, etc.), la estructuración de los portales mediante menús con categorias univocas, la eliminación de clicks innecesarios para llegar al lugar deseado, la disposición de buscadores y tablas dinámicas que permitan el filtrado de datos o de listados de expedientes, la disposición de documentos en varios formatos de uso común y reutilizables, la exposición de datos cuantitativos mediante gráficos explicativos, la comprobación de la vigencia de la información, entre otros muchos.

Así, la pauta generalizada es que los indicadores se limiten estrictamente a los tipos de información disponible, e incurren y retroalimentan el mismo error que se señala de manera generalizada respecto a los portales de transparencia y al cumplimiento de la publicidad activa. En esta línea, Martín Nájera (2018: 25) describe la situación ante aquellos portales que "aun cumpliendo la letra de la Ley", originan problemas prácticos que limitan cualquier funcionalidad, como no permitir la exportación de datos o el filtrado de la información a través de unos descriptores mínimos para localizar la información. Son portales de transparencia para aparecer como transparentes, pero sin que sea una transparencia aplicada al ciudadano.

Por ello, la principal carencia de las mediciones deriva de su grado de profundidad, que debería ser mucho mayor, lo que por otro lado puede provocar su inviabilidad por los recursos que sería necesario aplicar a esta finalidad. Por el contrario, abundan las mediciones que buscan contrarrestar esa necesidad de dedicación y optan por la simplicidad, aun a costa de captar solamente la transparencia formal y no real. Una fórmula habitual es recurrir a indicadores dicotómicos (si/no, cumple/no cumple) que son fáciles de aplicar, aunque no permiten captar una gradación. Por otra parte, las mediciones que aplican gradaciones no siempre tienen reglados claramente los criterios que permiten puntuar de un modo u otro en cada indicador.

También se utilizan metodologías que invierten la carga del esfuerzo a las entidades objeto de medición, al solicitar que se autoevalúen, de manera que solo haya que verificar la información obtenida. Esta práctica es cada vez más frecuente y ya comienza a acusar el cansancio de muchas entidades objeto de medición, que se ven compelidas a cumplimentar cuestionarios para mediciones que no en todos los casos son oficiales y sobre las que no siempre hay conformidad respecto a sus criterios, viéndose obligadas ante la inquietud de que la no colaboración quede plasmada públicamente como un indicio de falta de transparencia. Este tipo de 


\section{Javier Sierra Rodríguez}

autoevaluaciones muestran defectos típicos como dar por bueno el señalamiento de supuestos cumplimientos porque se indican URLs donde efectivamente se aloja una información, la cual sería imposible de localizar para cualquier ciudadano desde la página principal del portal. También hay organizaciones que, una vez que conocen los indicadores, se adaptan rápidamente para cumplir con el mínimo que se va a medir, dejando olvidados el resto de contenidos que deberían corresponder a una entidad transparente.

\subsection{Consecuencias derivadas.}

Las expectativas ciudadanas sobre las mediciones de transparencia se enfrentan a los bajos niveles de confianza que existen sobre cualquier actuación que se asocie a la actividad pública, más aún, ante una ciudadanía que ha mostrado su descontento de manera intensa en los últimos años. En este marco, las escasas referencias a las mediciones que se pueden encontrar en encuestas de opinión pública nos llevan al Barómetro de la Transparencia de España, que en el año 2016 incluyó dos preguntas sobre el conocimiento de las mediciones de transparencia y su credibilidad.

Según los datos de la encuesta, un 53,4\% afirmaba no haber oído nada sobre ellas, mientras que un $11,4 \%$ creía no tener noticias al respecto, es decir, que un $64,8 \%$ de los encuestados (dos de cada tres), parece que no tenía constancia. Por el contrario, prácticamente uno de cada tres encuestados si había oído sobre ellas (21,8\% sí y $12,8 \%$ creo que sí).

\section{¿Ha oído hablar alguna vez de las mediciones y rankings que puntúan a las organizaciones en función de su nivel de transparencia? (\%)}

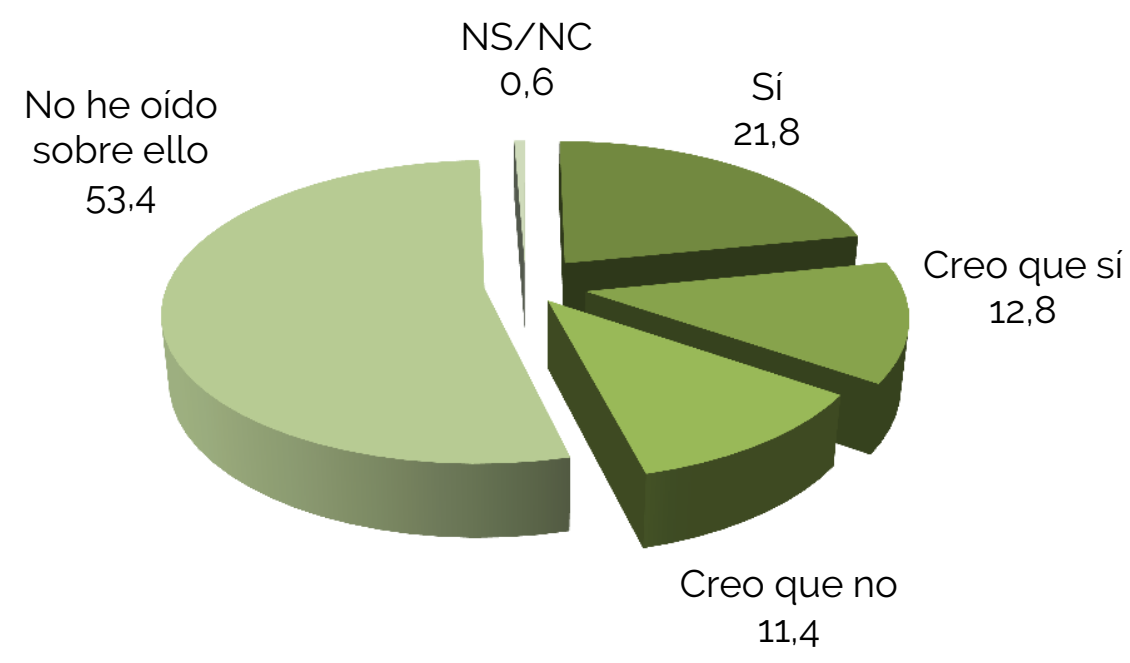

Fuente: Asociación de Profesionales de la Transparencia acreditra.com 


\section{Mediciones y premios de transparencia}

A quienes habian respondido afirmativamente, se les preguntaba por la credibilidad de las mediciones y rankings, obteniendo que el 59,5\% les otorgaba poca (43,6\%) o ninguna (15,9\%) credibilidad; mientras que el 30,8\% les daba bastante credibilidad y el $7 \%$ mucha.

\section{¿Qué credibilidad le merecen dichas mediciones y rankings? (\%)}

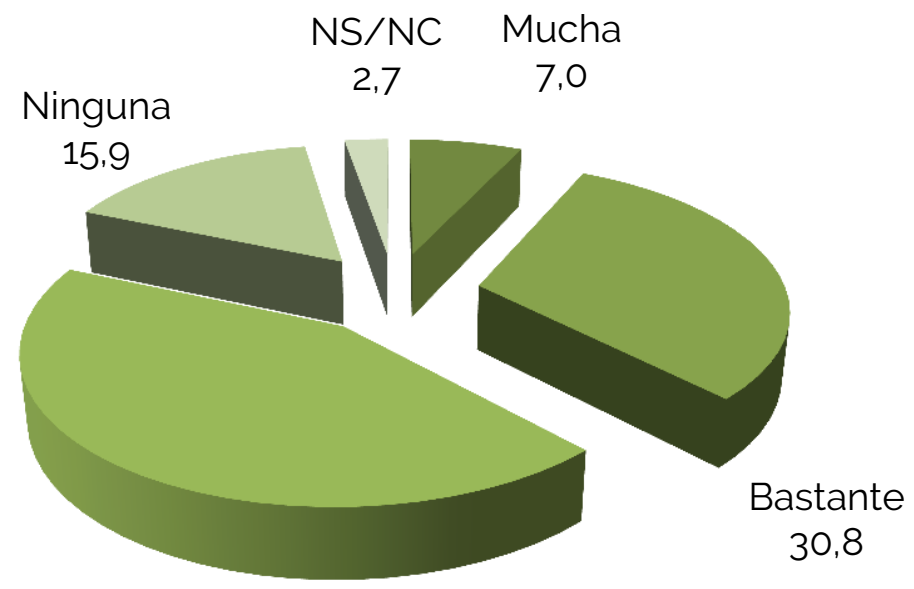

Poca

43,6

Fuente: Asociación de Profesionales de la Transparencia acreditra.com

De estos datos deriva la necesidad de que los sistemas de medición sean especialmente cuidadosos y busquen tener una robusta credibilidad. Sin embargo, debido a los problemas descritos y a la coexistencia de las mediciones basadas en metodologías dispares, resultan situaciones como el hecho de que aparezca una misma entidad en posiciones sustantivamente distintas en diferentes rankings. En un ejercicio comparativo realizado por Delgado Morales (2015) con el ITA y del Test de Aplicación Municipal de la Ley de Transparencia de 2014, en el que se comparaban los resultados de 108 Ayuntamientos incluidos en ambas mediciones, los resultados mostraban amplias oscilaciones con entidades que aparecian al mismo tiempo entre las primeras y últimas posiciones ${ }^{19}$.

Por ello, el autor indicaba que el cumplimiento de los indicadores no tiene por qué garantizar "que las entidades sean objetivamente transparentes", algo que se sustenta por la disparidad de resultados según la metodología aplicada, de modo que el método puede ser el que ocasione las variaciones en la posición. No

19 Entre los casos más extremos estaba el Ayuntamiento de Ciudad Real que obtenía la posición 25 en el ITA y la 107 en el Test (una variación de 82 puestos), y de Elche, en la posición 102 del ITA y la 14 del Test (una variación de 88 puestos). 


\section{Javier Sierra Rodríguez}

obstante, también hay muchas coincidencias entre los rankings, y aparte, hay que guardar cierta cautela porque es posible avanzar rápidamente en materia de publicidad activa gracias al dinamismo que permiten las páginas web.

Ante la situación descrita, se observa que cuando los técnicos de las organizaciones evaluadas ven cambios drásticos de posición, de su organización o de otras con las que ellos mismos se comparan, es fácil que adopten una postura defensiva frente a la metodología o seriedad de las mediciones. Pero además, en un plano más general, cuando los resultados de los rankings tienen un amplio impacto en medios de comunicación y aparecen simultáneamente entidades públicas distintas como las más transparentes, existe un riesgo de descrédito y de causar un perjuicio para todos aquellos que trabajan en torno a la promoción de la transparencia.

Aparte, el hecho de centrar las metodologías de medición en la publicidad activa, hace que podamos imaginarnos lo que debe pensar el ciudadano de a pie que no haya tenido una respuesta a su solicitud de acceso, mientras que esa entidad aparece como la más transparente. Es contraproducente que destaquen como transparentes las entidades que más dilatan las respuestas, mantienen respuestas esquivas, deniegan el acceso mediante interpretaciones forzadas de las causas de inadmisión y los límites, o simplemente optan por el silencio administrativo ante solicitudes de acceso a la información. Pero más aún, limitándonos a la vertiente de la publicidad activa, se da por hecho que los resultados de las mediciones no tienen por qué corresponderse con la percepción que puedan tener los ciudadanos, entre otros motivos porque sus metodologías no están diseñadas para ello: muchos ciudadanos buscarán información y no la encontrarán, preguntándose como una entidad muy transparente ha podido llegar a serlo.

La debilidad de la mayoria de las mediciones invita a que se produzcan con mayor intensidad los procesos que concentran sus esfuerzos solo en aquello que se mide, de manera que los indicadores -tal y como se ha visto- pierden su eficacia para captar la realidad, originándose procesos de aprendizaje perverso (Meyer y Gupta, 1994). Como consecuencia, se olvida todo lo que no es objeto de medición, de manera que los rankings pueden estar liderados por entidades cuyos portales de transparencia sirven solo para eso, para cumplir los indicadores, pero nada más, de modo que las páginas web de publicidad activa en lugar de convertirse en portales para el ciudadano, sean meros portales de cumplimiento de la publicidad activa a tenor de los rankings.

Garrido y Zafra (2017: 90) describen esta situación al afirmar que hay portales que ni siquiera se habian concebido para cumplir con las estipulaciones que establece la legislación en materia de publicidad activa, sino "para cumplir con dicho índice exclusivamente". Esta realidad se sigue corroborando, al observar portales que sin ningún tipo de reparo incluyen los logotipos de las entidades que realizan las 


\section{Mediciones y premios de transparencia}

mediciones, y que hacen constar como índice de contenidos la relación de bloques e indicadores originales de dichas mediciones, que completan con enlaces a documentos PDF, constituyendo repositorios documentales no orientados al ciudadano ${ }^{20}$. Dicho de otro modo, portales zombis a los meros efectos de ser bien puntuados en los rankings que no se detienen a valorar aspectos cualitativos o relacionados con la calidad y presentación de la información (Sierra y Soto, 2019: $760)^{21}$.

En cualquier caso, no tendría sentido hablar de los problemas de este cumplimiento formal de los indicadores si no hubiese algún tipo de premio o recompensa a este tipo de conducta. Como ya se mencionó, la transparencia se considera un valor positivo y el calificativo de transparente es objeto de ambición por los responsables de la transparencia en cada entidad, pero especialmente por la esfera política que desea asimilar a su gestión el calificativo de transparente y que no dudará en utilizar de manera propagandística cualquier medalla o premio que le otorgue un ranking de transparencia.

\section{REFLEXIÓN FINAL}

El escenario actual de las mediciones de la transparencia parece no ser el más adecuado para la finalidad de dotar de credibilidad a los avances en materia de transparencia. La diversidad de mediciones con metodologías diferentes muestran resultados dispares y pueden conllevar el descrédito del trabajo que se está desarrollando y que afecta, no solo a las entidades que miden la transparencia, sino a todos los que trabajan en esta materia.

Por este motivo, los profesionales, expertos, académicos y técnicos, así como las organizaciones a las que pertenecen - públicas y privadas- están legitimadas, no solo para dar su visión crítica sobre la situación, sino también para demandar activamente que se establezca algún tipo de estándar mínimo para no lanzar mensajes contradictorios a la sociedad.

En esta línea, no hace falta justificar más las razones por las que debería ser el CTBG, el organismo que debería tomar la iniciativa para que se ponga en marcha un sistema reconocido y de medición más profunda de todas las vertientes de la

\footnotetext{
20 Además, se pueden detectar casos de portales completamente desactualizados que en algún momento han podido responder a inercias concretas para aparecer en posiciones superiores de los rankings, pero que posteriormente han sido abandonados. Un peligro adicional es que los portales eliminen información considerada sensible o incómoda una vez superado el proceso de evaluación correspondiente.

21 Delgado (2016: 3) es aún más crítico y hace referencia a la transparencia estética "que esconde en su interior la trampa de la opacidad de los datos en bruto y que utiliza la infoxicación como herramienta de ocultación, que se llena la boca de transparencia sin aportar información real y útil, o para referirse a una mera pantomima que tiene como objetivo más parecer transparente que serlo realmente".
} 


\section{Javier Sierra Rodríguez}

transparencia. El CTBG es el organismo de control madre, que podria distribuir las tareas de medición en colaboración con los Consejos y Comisionados autonómicos -cada uno atendiendo a su singularidad y atribuciones posibles-, para que cada entidad asuma las correspondientes a su ámbito territorial, todo ello sin olvidar los mecanismos de cooperación que se pueden establecer con las entidades de la sociedad civil.

En un escenario ideal, el sistema a implementar mediría qué se publica, pero también cómo se publica, con valoraciones que se fijen especialmente en la calidad de la información y de su presentación. No se ceñiría a reflejar las obligaciones de la ley, sino también los indicadores de transparencia que fuesen más allá según lo que en cada momento se considere que constituye un mínimo a cumplir. No se limitaría a la publicidad activa, sino que incorporaría el examen real de la gestión y resolución de las solicitudes de acceso a la información. A su vez, debería combinar el enfoque de auditoría, es decir, la comprobación externa de indicadores; con la experiencia de los ciudadanos, considerando su percepción y valoración tras la visita a las páginas con información pública o tras la solicitud de acceso a una determinada información. Este tipo de mediciones se acercarian más a lo que se entiende por evaluación, concepto que se ha tratado de evitar deliberadamente a lo largo de este trabajo, porque simples mediciones de indicadores no deberian asociarse a ejercicios de evaluación que son más complejos y completos. Existen modelos destacables, como la metodología MESTA, con muchas de las virtudes mencionadas, pero que carece de lo más importante: su aplicación generalizada. También hay experiencias a imitar y aprovechar, como las innovaciones metodológicas que ha puesto en marcha el Sindic de Greuges en Cataluña y los desarrollos tecnológicos del Comisionado de la Transparencia de Canarias.

Sin embargo, la cantidad de recursos que sería necesario disponer para hacer este tipo de mediciones y extenderlas sobrepasa las posibilidades actuales. La Declaración de Cádiz de 2018 de los Consejos y Comisionados de Transparencia denunciaba la escasez de medios con la que se dota a los organismos de garantía. Es necesario su refuerzo para el desempeño de estas y otras funciones, pero aun así, no sería posible extender completamente las mediciones propuestas por el número tan elevado de organizaciones que existen. Como solución se podrian abarcar, al menos, las principales entidades de carácter público y las que son de referencia: AGE e instituciones del Estado, Comunidades Autónomas, Ayuntamientos de más de 50.000 habitantes, entre otras.

Este enfoque no supone desplazar el trabajo que desempeñan entidades decanas como Transparencia Internacional, la Fundación Compromiso y Transparencia, o los grupos de investigación de las Universidades. Al contrario, en un escenario ideal, se trataría de integrar el trabajo que desarrollan estas organizaciones mediante los correspondientes protocolos de colaboración o articulación de un 


\section{Mediciones y premios de transparencia}

trabajo en red. Para ello, lo ideal sería que el CTBG hiciese de director de orquesta, promoviendo las líneas de mejora, evitando la duplicidad de mediciones e impulsando una ordenación -ni necesariamente, ni deseablemente jurídica- del ámbito de medición de cada organización. De hecho, cualquier sistema de estas características debería contemplar algún tipo de grupo de trabajo o comisión de control externo, que vele por su perfeccionamiento y por la detección de problemas, en el cual deberian estar representadas las entidades que han demostrado su solvencia técnica y profesional y que disponen de experiencia acumulada en este ámbito.

En cualquier caso, la disyuntiva actual pasa por no hacer nada y dejar abierto el riesgo a la banalización de cualquier calificación o premio de las entidades que se presumen como más transparentes, o poner este tema como una prioridad de la agenda de los organismos de garantía. A su vez, pasa por decidir el modelo que queremos, si tener -como hasta ahora- muchas mediciones susceptibles de mejora, o bien, tener pocas y muy buenas.

El reto planteado es de muy difícil consecución a corto plazo, por lo que este trabajo tiene como expectativa que sirva para la reflexión y para que las organizaciones que actualmente desarrollan mediciones dispongan, en un solo documento, de las principales críticas y puntos débiles que se observan, de manera que puedan mejorar su diseño y su método de captación de la realidad. De hecho, cualquier esfuerzo en esta linea es un trabajo a desarrollar paulatinamente y, aun así, siempre quedará algo que mejorar porque las exigencias respecto a la transparencia se espera que sigan evolucionando.

Finalmente, cabe apuntar que son muchas las voces que esperan de los organismos de control un pronunciamiento extenso sobre la situación de las mediciones de la transparencia, destacando las buenas prácticas y explicitando los aspectos que deben ser corregidos a la hora de elaborar y publicar mediciones y valoraciones sobre la transparencia. 


\section{Javier Sierra Rodríguez}

\section{BIBLIOGRAFIA}

Abellá, A., Ortiz de Urbina Criado, M. y De Pablos Heredero, C. 2014. "Meloda, métrica para evaluar la reutilización de datos abiertos". El Profesional de la Información, vol. 23, núm. 6:582-588

http://Www.elprofesionaldelainformacion.com/contenidos/2014/nov/O4.pdf

Arízmendi, E. 2017. "Metodología de Evaluación y Seguimiento de la Transparencia de la Actividad Pública (MESTA)", en Revista Española de la Transparencia, núm. 4:40-44.

Alonso Magdaleno, M.L. y García-García, J. 2014. "Evaluación de la Transparencia Municipal en el Principado de Asturias". Auditoria Pública, 64, 75 - 86.

Barqueros Sánchez, I. 2018. "Transparencia en la gestión/explotación de redes de transporte público. El caso de la Comunidad de Madrid", en Serrano Maillo, I., Aplicación de la transparencia: educación y universidad, seguros, medioambiente, justicia y contratación. Madrid: UCM, págs. 159-172.

Caamaño Alegre, J., Lago Peñas, S., Reyes Santias, F. y Santiago Boubeta, A. 2011. "Budget transparency in local governments: an empirical analysis", Internacional Studies Program Working Paper Series, 11-02:1-30.

Gálvez Rodriguez, M.M., Caba Pérez, M.C. y López Godoy. M. 2009. "La transparencia online de las ONGs españolas". Revista Española del Tercer Sector, núm. 13: 63-86

García García, J., Alonso Magdaleno, M.I. y Alonso Magdaleno, M.L. 2016. "Determinantes de la transparencia en municipios de mediano y pequeño tamaño", Auditoría Pública, 67:51-60.

Garcia Garcia, J. y Curto Rodríguez, R. 2018. "Divulgación de información pública de las Comunidades Autónomas Españolas (2013-2017): portal de datos abiertos, portal de transparencia y web institucional", El Profesional de la Información, vol. 27. núm. 5 .

Beltrán Orenes, P. y Martínez Pastor, E. 2016. "Grado de cumplimiento de las leyes de transparencia, acceso y buen gobierno y de reutilización de los datos de contratación de la administración central española", El Profesional de la Información, vol. 25, núm. 4: 557-567.

De la Fuente, G. 2015. Modelo teórico de medición de la transparencia para la RTA. Documento de Trabajo de la Red de Transparencia y Acceso a la Información.

Delgado Jalón, M.L., Navarro Heras, E. y Mora Agudo, L. 2017. "Cumplimiento de los requisitos de transparencia: un diagnóstico de la situación para los municipios españoles de más de 50.000 habitantes", Innovar, vol. 27, núm. 66:109-121.

http://dx.doi.org/10.15446/innovar.v27n66.66806 


\section{Mediciones y premios de transparencia}

Delgado Morales, F. 2015. "Sistemas de medición y Transparencia en los Ayuntamientos". Revista Española de la Transparencia, núm. 0: 12-17;

Delgado Morales, F. 2016. "La Transparencia de Shródinguer". Revista Española de la Transparencia, núm. 3: 3-4;

Delgado Morales, F., López Carvajal, F.J. y Sierra Rodríguez, J. 2015. "Regulación y sistemas de evaluación de la transparencia", Revista de Derecho de la Hacienda Pública, núm. 5: 111-134.

Díez Garrido, M. 2017."La transparencia del Partido Popular y Ciudadanos en sus sitios web. Análisis de las campañas electorales de 2015 y 2016", Dígitos, núm. 3:115-132.

Esteller More, A. y Polo Otero, J. 2012. "(Why) does your local government respond?", Public Management Review, Vol.14:1153-1173. http://dx.doi.org/10.1080/14719037.2012.657839

Garcia Melián, J.C. 2017. "Control de la transparencia: baremos y acreditación", en Sánchez de Diego Fernández de la Riva, M. 31 visiones de la transparencia en España. Madrid: UCM, pp. 324-340.

Garrido Rodríguez, J.C. y Zafra Gómez, J.L. 2017. "Evaluación del nivel de transparencia municipal. Aplicación al caso andaluz". Auditoría Pública, 69:85-94.

Giménez-Chornet, V. 2017. "La auditoría en la gestión de la transparencia del sector público", Métodos de información, núm. 8(15): 111-123.

Jiménez Asensio, R. 2017. "Instituciones de garantia de la transparencia", El Cronista del Estado Social y Democrático de Derecho, núm. 68, 2017: 60-75.

Manfredi Sánchez, J.L., López Cepeda, A.M., Álvarez Peralta, M., Saiz Echezarreta, V. y Reig Cruañes, J. 2016. "Transparencia y rendición de cuentas de los municipios de Castilla La Mancha", Barataria, núm.21:175-189. https://doi.org/10.20932/barataria.voi21.304

Martín Delgado, I. 2018. "La configuración legal de los consejos autonómicos de transparencia: algunos riesgos para el ejercicio de su función de control", Dilemata, Revista Internacional de Éticas Aplicadas, n 27, 281-309

Martín Nájera, S.A. 2018. "Zonas oscuras de la transparencia", Auditoría Pública, núm. 71:23-32.

Martínez Isidoro, B. 2017. "Estudio comparado de la transparencia de los partidos políticos en el Congreso de los Diputados de las Legislaturas X y XI", en Sánchez de Diego Fernández de la Riva, M. (Coord.), 31 visiones actuales de la transparencia en España. Madrid: UCM. 


\section{Javier Sierra Rodríguez}

Meyer, M.W. y Gupta, V. 1994. "The performance paradox". Research in Organizational Behaviour, núm. 16, 309-369.

Molina Rodríguez-Navas, P., Rodríguez Breijo, V., Corcoy Rius, M. y Vadillo Bengoa, N. (2017). "La transparencia de los ayuntamientos de Cataluña antes de la entrada en vigor de la legislación estatal y autonómica". Anales de Documentación, vol. 20, núm. 2, 1-11.

Molina Rodríguez-Navas, P; Simelio Solà, N.; Corcoy Rius, M. 2017. "Metodologías de evaluación de la transparencia: procedimientos y problemas", Revista Latina de Comunicación Social, 72:818-831. Disponible en: http://WwW.revistalatinacs.org/o72paper/1194/RLCS-paper1194.pdf

Mora, L., Delgado, M.L. y Rivero, J.A. 2018. "Influencia del marco normativo en la transparencia: análisis de los ayuntamientos españoles, 2014-2017", Revista Española de Ciencia Política, núm. 48:207-255.

Moreno Sardá, A.; Molina Rodríguez-Navas, P.; Corcoy Rius, M.; Aguilar Pérez, A.; Borràs Farran, M. 2013. "Infoparticip@: periodismo para la participación ciudadana en el control democrático. Criterios, metodologías y herramientas", Estudios sobre el mensaje periodístico, Vol. 19, Núm. 2:783-803.

Moreno Sardà, A.; Molina Rodríguez-Navas, P.; y Simelio Solà, N. 2017. "Impacto de la legislación sobre la transparencia en la información publicada por las Administraciones Locales". El profesional de la Información, vol. 26, núm. 3:370380 .

Pintado Pérez, J. 2018. "Aproximación a un modelo de transparencia para los partidos politicos", en Sánchez de Diego Fernández de la Riva, M. (Coord.) 2018. Aplicación sobre la Transparencia. Madrid: UCM, págs. 165-178.

Tejedo Romero, F. y Ferraz Esteves de Araujo, J.F. 2015. "Determinants of Local Governments' Transparency in Times of Crisis: Evidence From Municipality-Level Panel Data", Administration \& Society, Vol. 50: 527-554.

Rey Jordán, D. 2016. "La transparencia como motor de la innovación pública y base para el gobierno abierto", Actualidad Administrativa, núm. 10.

Ros Medina, J.L. 2018. "La evaluación de la Transparencia en España a debate metodológico: MESA e Índices de Transparencia Internacional". Revista Internacional de Transparencia e Integridad, núm. 6:1-22.

Sánchez de Diego Fernández de la Riva, M. 2014. "El día después de la ley de transparencia", en Revista Juridica de Castilla y León, núm. 33: 1-20.

Sánchez de Diego Fernández de la Riva, M. (Coord.) 2018. Apuntes sobre la Transparencia. Madrid: UCM.

Scrollini, F. 2012. Evaluación de la Transparencia: Estado del Arte e indicadores. Santiago de Chile: Consejo de la Transparencia. 


\section{Mediciones y premios de transparencia}

Sendín García, A. 2014. "El Consejo de Transparencia y Buen Gobierno", Revista Jurídica de Castilla y León, núm. 33. Monográfico, Transparencia, Acceso a la Información y Buen Gobierno.

Sierra Rodriguez, J. 2014. "Certificación en transparencia: el sistema español de acreditación de la transparencia", Revista Más Poder Local, 21:28-32.

Sierra Rodríguez, J. 2018. "Una crítica al diseño de los organismos de garantía del derecho de acceso en las Comunidades Autónomas", Revista Juridica de Castilla y León, núm. 45.

Sierra Rodríguez, J. y Soto García, M. 2019. "Las Administraciones Públicas de Andalucía y el cumplimiento de la legislación estatal y autonómica de transparencia pública y acceso a la información", en Troncoso Reigada, A., Transparencia Pública y Comunidades Autónomas. Valencia: Tirant Lo Blanch, pp. 751-803.

Villoria Mendieta, M. e Iglesias Alonso, A. 2017. "Developments In Transparency In The Spanish Municipalities: An Analytical Revision Of The Last Research", Lex Localis, Vol. 5. Núm. 3: 411-432.

Viñas Xifra, J. 2018. "La transparencia y el control en el sector público: ¿hacia un cambio en la auditoria pública local?, Auditoría Pública, núm. 71: 33-40.

Zafra Gómez, J.L. y Garrido Rodríguez, J.C. 2017. Una propuesta metodológica para medir la transparencia en el ámbito municipal. Sevilla: Cámara de Cuentas de Andalucia. 